\title{
8-31 Generating Facial Images Eye-Contacting with Partner on the TV Conference Environment
}

\author{
Tsuyoshi Yamaguchi $^{\dagger}$ \\ SCCS, \\ Chukyo University
}

\author{
Masafumi Tominaga \\ SCCS, \\ Chukyo University
}

\author{
Kazuhito Murakami ${ }^{\mathrm{t}}$ \\ Faculty of I. S. T.. \\ Aichi Pref. University
}

\author{
Hiroyasu Koshimizu \\ SCCS, \\ Chukyo University
}

\begin{abstract}
We have been developing the method for facial part recognition such as eyes, nose, mouth, etc. This paper pays attention especially to eye (iris) recognition by Hough transform.

Basing on this iris recognition, this paper proposes a basic idea and its method for re-generation of facial image which is eye-contacting with partner on the TV conference environment. Through this we propose a new methodology to realize a natural human interface of the face-to-face communication on the TV conference environment.

Since human is likely to look at the face of his partner on TV monitor not at TV camera, he will usually fail to send his own eye-contacted facial images to him. The basic idea of this paper to improve this fatal communication degradation is to regenerate facial image by changing the direction of the irises in the same original facial imagc. The proposed methods and some experimental results are presented to demonstrate the effectivity and the coming inherent subjects.

Future problems are to improve the regeneration method of irises, to introduce the real time processing method, to cope with the motion of the head and finally to propose a new eye-camera system which could reduce the constraints caused by the head mouted goggle.
\end{abstract}

\footnotetext{
'Address: 101 Tokodate, Kaizu-cho, Toyota 470-0393 JAPAN. E-1ail: \{tsuyoshi@koshi-lab., hiroyasu@\}sces. chukyo-u.ac.jp

Address: Kumahari, Nagakute-cho, Aichi-gun. 180-1198 JAPAN, I-mail: murakami@ist.aichi-pu.ac.jp
}

\section{Introduction}

In usual cases, we can't sufficiently watch the eyes of our partners in the TV conference environment since our partner's face is displayed on the TV monitor not on the TV camera which is looking at our faces. Even in the case of the hand language communication, it is well known that almost all visual interests are focused on the face. ${ }^{[8]}$ This typical example suggests us a very important problem of human interface inherent at everywhere of the newly coming cyber social environments. Therefore, since human is likely to look at the face of his partner on TV monitor not at TV camera, he will usually fail to send his own eye-contact facial images to him. This paper is intended to cope with this essential problem. The basic idea of this paper to improve this fatal communication situation is to regenerate facial images by changing the direction of the irises in the same original facial images. Although many researches on facial image processing ${ }^{11}$ have been reported, there is no direct method to cope with this program in a similar way.

From almost the same aspect, some researches have been reported. Nakajima ${ }^{|5|}$ proposed a method for correcting the direction of the head to the TV monitor by using stereo matching method. Useless to say, this method can not provide any improvement to the eye-contact human interface. As well known from vision psychology ${ }^{[7]}$, in the traditional method, it is not always comfortable for us to use half-mirror system between TV monitor/ camera and us as a human interface tool. And this system is relatively expensive and is not good in utility for the natural human interface. Therefore we can't find no same research until now. In this way, this paper will porvide an unique technology for providing this kird of new human interface environment. 
Figure 1(a) shows the basic status for the necessity of this research. Although the person wants to put his eyes to contact to his partner on the monitor, his eye glance is likely to be out of the eye contact for his partner as shown in Fig.l (b). In this paper, we propose a method for generating the eye-contacted facial image by changing the direction of the irises as shown in Fig. I (c).

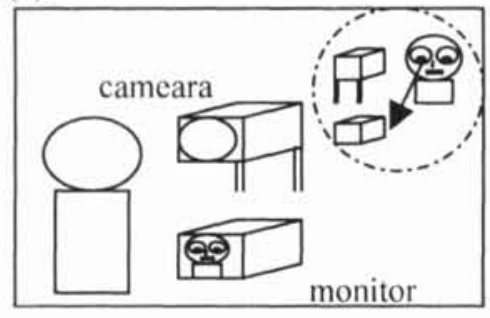

(a). TV conference

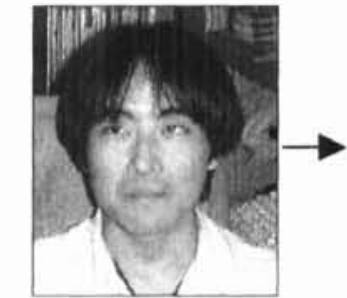

(b). Out of eye contacting

facial image

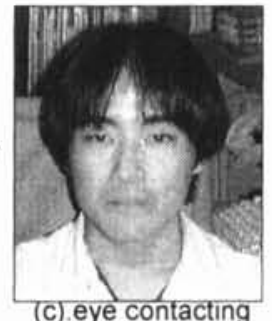

facial image
Fig. I Facial interface in TV conference

In Chapter 2, a method for extracting face and facial parts form the successive facial images is proposed by using image processing. Especially in Chapter 3, a method to recognize a pair of irises is developed by using Hough transform for circle detection. In Chapter 4, a paradigm to regenerate a pair of irises in the successive facial images is introduced in terms of the simple geometrical relationship among TV monitor, camera and testec. In Chapter 5, the successive methods to realize this idea by using image processing are proposed. The methods to segment white/black and eye-brim regions, to regenerate irises in the eye region of the gray facial image, and to control the direction of the irises are described in detail also with the respective experimental results.

\section{Extraction of Face}

Facial region is detected by using the projection of the binary image which is binarized by the thresholdint from the inpul gacial image fat $e^{|4|}$. In the vertica? projection histegram curve, a pair of the locations of tio first sharp changes is detected as the facial right and left positions. In the horizontal projection curve, a pair of the locations of the first sharp changes is detected as the facial top and bottom positions. Preprocessing was especially developed by using the properties of the facial image.

\section{Recognition of Irises by Hough Transform}

A method for recognition of irises from the gray images is proposed by using Hough transform for circle detection. Several candidates of a pair of the irises were extracted at first by applying Hough transform for circle detection to the binary image. The binarization method was especially realized by [1],[4]. The voting ranges of the parameter space $(a, b, r)$ were limited to some extent in order to reduce the computation cost and to enforce the performance. Parameters a and b indicate the center of iris. Parameter $r$ indicates the radius of the iris. The best pair of the irises is detected from the candidates in accordance with the criteria standards, that the positional relation between left and right irises is horizontal and that the radius of the left equals to the right. Therefore the recognition procedure was prepared as follows:

Step 1. Set $K$ as the threshold for the peak detection.

Step 2. Detect the coordinates of irises whose peak is greater than or equal to $\mathrm{K}$.

Step 3. Prepare the list of all candidates of the irises.

Step 4. Choose the candidates pairs whose vertica! diatance is smaller than the threshold

Step 5. Choose the candidates pairs from the list whose radius are equal.

Step 6. Among the rest of the list, extract a pair of right and left irises whose horizontal distance is minimum.

The robustness of this algorithm was $97 \%$ by using 30 facial images, and consequently it was known that the facial parts regions could be extracted based on the location of the irises over $95 \%$ recognition rate at worst by using 96 facial images. Figure 3 shows an example $e^{[1] \cdot[4]}$. 


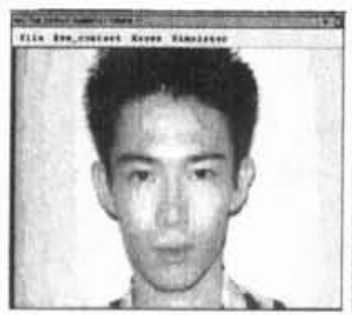

(a) input face

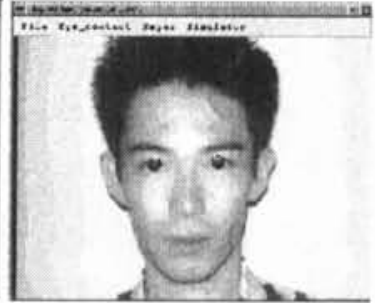

(b) recognition of irises
Fig. 3 Example of the extracted irises

\section{Model of the Eye Contact}

In order to model the situation of the TV conference environment shown in Fig. I(a), the parameters $\mathrm{R}$ and $\mathrm{r}$ are specified for modeling the vertical relation between the TV camera and the monitor, and the parameter $\mathrm{L}$ is specified for the horizontal relation between them. This is depicted in Fig.4.

In the beginning, let us imagine the iris moves from the coordinate $\left(\mathrm{x}_{0}, \mathrm{y}_{0}\right)$ extracted before to the new coordinate $\left(x_{1}, y_{1}\right)$. This new coordinate $\left(x_{1}, y_{1}\right)$ can be easily calculated by eq.(1) characterized with the parameters $\theta$ and $\theta_{2}$ indicating the spacial relationship among a person, camera and monitor.

In this expression, functions $\Delta \mathrm{x}$ and $\Delta \mathrm{y}$ are designed to convert the parameter $\theta$ to the number of the pixels in the facial image.

$$
\begin{aligned}
& x_{1}=x_{0}+\Delta x(\theta): \Delta x(\theta)=\theta / 10 \\
& y_{1}=y_{0}+\Delta y\left(\theta_{2}\right): \Delta y\left(\theta_{2}\right)=\theta_{2} / 10
\end{aligned}
$$
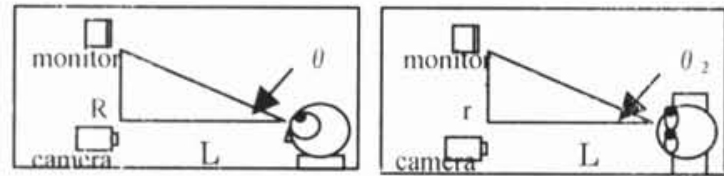

Fig. 4 Spacial relationship among threc objects

\section{Regeneration of Eye-Contacted Facial Image}

\subsection{Segmentation of the eye region}

The center coordinate $\left(\mathrm{x}_{1}, \mathrm{y}_{1}\right)$ and the radius $\mathrm{rl}$ of the extracted iris are utilized to regenerate the moved iris. Beforehand the eye regions are recognized by utilizing the extracted eye center coordinate $\left(\mathrm{x}_{0}, \mathrm{y}_{0}\right)$. The eye regions(the white of eye, iris, skin and contour of the eyelid) are extracted for both of eyes, and basing on the recognition results, the moved iris is generated within the contour of the eyclid. Figure 5 show the procedures for recognizing and regenerating irises.

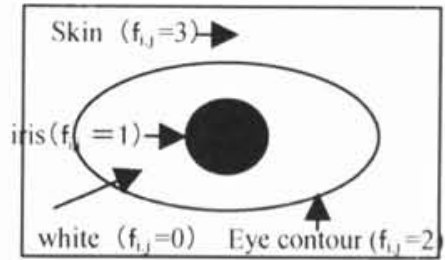

Fig. 5 The white of the eye

\section{2 Regeneration of the irises}

The pixels $(x, y)$ in the region for new iris are painted in black at the region where the distance $\mathrm{d}$ between $\left(\mathrm{x}_{1}, \mathrm{y}_{1}\right)$ and $(\mathrm{x}, \mathrm{y})$ is less than or equal to the radius do. All pixels $(x, y)$ within the contour of the eyelid are painted in white at the region where the distance is greater than or equal to $\mathrm{r} 1$. The black and white colors are decided as follows:

black $=\min \{F i j \mid$ fij $=1\} \quad$ white $=\max \{F i j \mid$ fij $=0\}$

where Fij : gray value and fij : label value

After this procedure, the final irises are regenerated by smoothing processing.

\section{Experiments and Applications}

\subsection{Realizing Eye-contacted TV conference environment}

We applied this system to the input facial image shown in Fig.6(a), and then new facial image given in Fig.6(b) was provided in such a way that the directions of the irises are slightly moved upward. Even though the details in the regenerated eyes given in Fig.6(b) seem not perfect, we can watch it naturally at the regenereted facial image shown in Fig.6(b) especially in the movie demonstration.

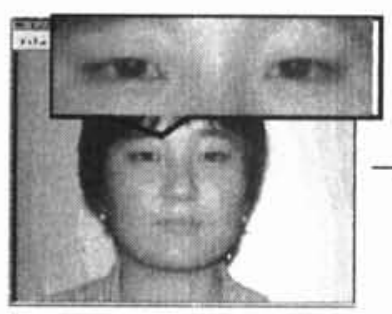

(a) input face

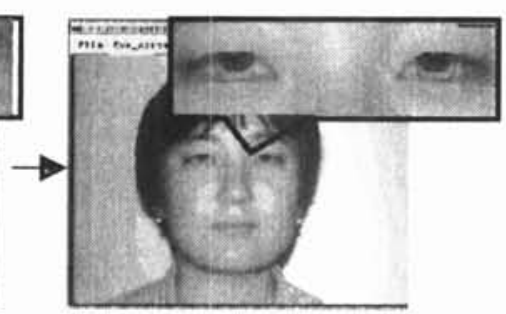

(b) regenerated facial image lig.6 Experimental result

\section{Eye-camera system which does not} restrain the testee

The principle of this Eye-Contact Camera system is comected with the eye camera which doc: not restrain the testee's head. At present, the eye camera with the 
head mouted goggles must be fixed on the head completely. Therefore, the operation is difficult in calibration etc. and the reliability of the eye-mark pattern becomes low. A new principle of eye-camera system which does not restrain the testee can be proposed by these image processing procedures.
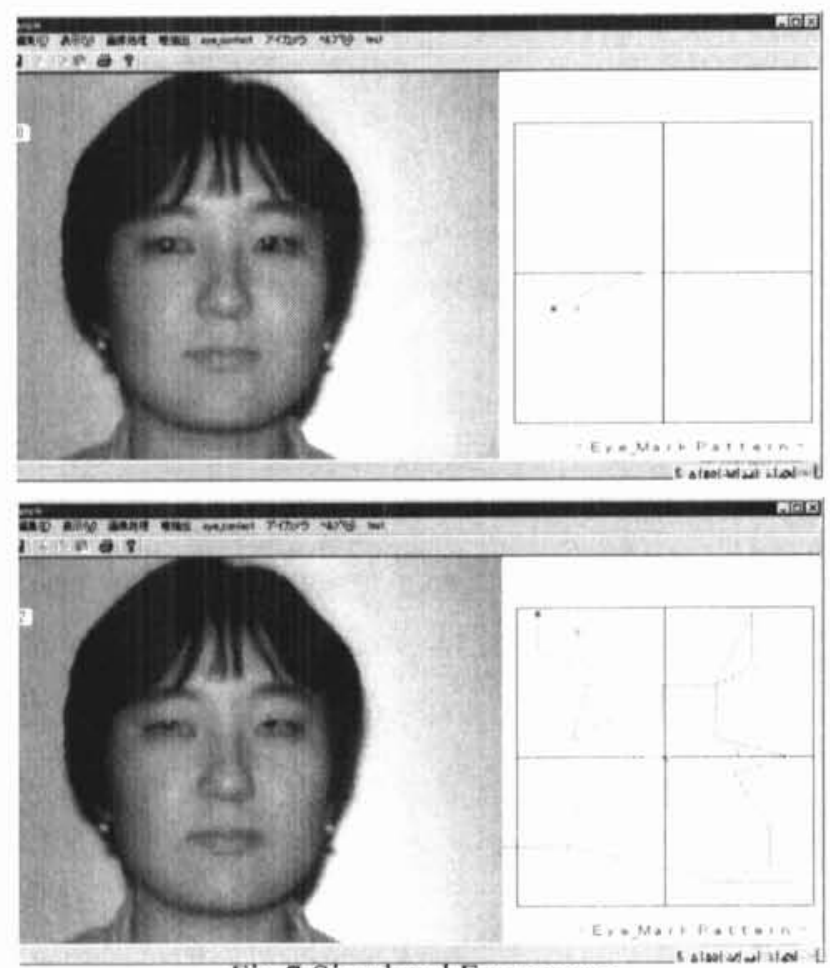

Fig.7 Simulated Eye-camera

Figure 7 shows an experimental result. In this experiment, the resolution power of around $\Delta x=$ $10 \mathrm{~mm}$ and $\Delta y=10 \mathrm{~mm}$ was realized by means of the facial image with the size $640 * 480$. This result was sufficient to acquire the visual information on where the testce is looking at among the facial parts in facial caricaturing system ${ }^{[10]}$.

\section{Conclusion}

In this paper, we have presented a method to recognize irises from the facial image, and we have shown the possibility for realizing eye-contact camera system. Future problems are to improve the regeneration method of irises, to introduce the real time processsing method and to cope with the motion of the head and finally to improve a new eye-camera system which could reduce the constraints caused by the head mouted goggle.

\section{Acknowledgments}

Authors wish to express thanks to IMS International HUTOP Research Project, Daiko Foundation Academic Research Promotion. Grant in Aid for Scientific Research Promotion of The Ministry of Education and HRC Research Promotion for supporting a part of this research.

\section{References}

[1] Oshida, M., Tominaga, M., Endoh. T., Toryu, T., Murakami, K., and Koshimizu. H. ; "Facial parts recognition by Hough Transform" . 1998 Annual meeting of Tokai-branch of IES, IEICE, D-708, P.354 (1998.9)

[2] Yamaguchi. T., Tominaga, M., Murakami, K., and Koshimizu. H. : "Generating Facial Images Eyc-Contacting with Partner on the TV Conference Environment" , Japan Academy of Facial Studies, I999 Forum Faces, B-4P, P.25(1999.8)

[3] Yamaguchi, T., Tominaga, M., Murakami, K., and Koshimizu. H. : "Generating Facial Images Eye-Contacting with Partner on the TV Conference Environment" . 1999 Annual meeting of Tokai-branch of IES, IEICE, G-745, P.373(1999.9)

[4] Segawa, Y., Endoh. T., Toryu, T., Murakami, K., and Koshimizu, II. : "Contour Extraction of face and irises for facial caricaturing " , Trans Image Information Media Society, Vol.51, No.1I.pp.1935-1944 (1997.11)

[5] Ueno, T., Andoh, S., and Nakajima, M. : "A Method of Making Front Face for Eye Contact in Teleconference System". The $5^{\text {th }}$ Symposium on Sensing via Image Information, SII'99, pp.7I $-76(1999.6)$

[6] Osamu Hasegawa, Shigeo Morishima and Masahide Kancko: Face Information Processing, Trans.IEICE , Vol.J80-A, No.8, pp.1231-1249(1997)

[7] Patrick Henry Winston: The Psychology of Computer Vision, MeGraw-Hill computer science serics(1975)

[8] Nagashima, Y. : Present State and Issues of SiLE ("Almost all interface media is face even in the hand gesture conversation." ), Invited talk, PRMU99-141

\section{(Nov. 19, 1999)(Okinawa)}

[9] http://www.bri.ntt.co.jp/info/kameg/emr-nc/ vesearches.html

[10] Tominaga, M., Fujiwara. T., Murakami. K.. and Koshimizu, II. : "On KANSE: I Facial Image Processing For Computerized Facial Caricaturing System PICASSO", Proc. SMC'99 (Oct 1999) (Tokyo) 